AperTO - Archivio Istituzionale Open Access dell'Università di Torino

\title{
Validation of the revised Food Neophobia Scale (FNS-R) in the Italian context
}

\section{This is the author's manuscript}

Original Citation:

Availability:

This version is available http://hdl.handle.net/2318/1669520

since 2018-06-11T11:10:20Z

Published version:

DOI:10.1016/j.appet.2018.06.004

Terms of use:

Open Access

Anyone can freely access the full text of works made available as "Open Access". Works made available under a Creative Commons license can be used according to the terms and conditions of said license. Use of all other works requires consent of the right holder (author or publisher) if not exempted from copyright protection by the applicable law. 
Validation of the revised Food Neophobia Scale (FNS-R) in the Italian context

\begin{abstract}
Measuring individuals' level of food neophobia, i.e., the reluctance to eat novel food, is a critical task since it negatively affects diet variety and quality. Using structural equations models, the revised Food Neophobia Scale (FNS-R) was validated with a sample of 711 Italian adults. After deleting 4 items characterized by both low face validity and a suboptimal association with the other items, and after correcting statistically for the acquiescent response-set, the resulting 6-item, fully balanced FNS-R showed a good construct validity. Moreover, it showed the expected positive correlations with General Neophobia and with Disgust Sensitivity. Finally, it resulted invariant across participants' genders, age classes, and levels of education, and across methods of administration (paper-and-pencil and on-line). Strong points and possible developments of the study are discussed.
\end{abstract}

Keywords: Food neophobia scale, general neophobia, disgust sensitivity, confirmatory factor analysis, structural validity, concurrent validity. 


\section{Validation of the revised Food Neophobia Scale (FNS-R) in the Italian context}

Market globalization, migration flows, and the spread of new lifestyles involving food have considerably increased the availability of novel foods in Western society. This historical and

structural evolution puts individuals at the crossroads between, on the one hand, trying these novel

foods and enlarging their eating repertoire, and on the other hand, limiting their consumption to

familiar foods. Psychologists have termed this latter orientation as food neophobia.

Food neophobia, the reluctance to eat unfamiliar foods, is a universal predisposition among humans and, more generally, omnivores (Rozin \& Millman, 1987; Rozin \& Vollemecke, 1986).

From an evolutionary perspective, each new food represents both an opportunity and a risk: the opportunity to expand the nourishment source set, but also the risk to ingest something dangerous or even life threatening. According to Rozin (1976), food neophobia arises from this 'omnivore dilemma.'

Notwithstanding the universality of food neophobia, there is room for inter-individual and intra-individual variability. Although serving a protective function in a potentially dangerous environment, in contemporary Western cultures characterized by high levels of food safety, food neophobia can be problematic, because it dramatically constrains individuals' food choices, limiting consumption variety and worsening diet quality (e.g., Siegrist, Hartmann, \& Keller, 2013; Skinner, Carruth, Bounds, \& Ziegler, 2002). Therefore, it is important to measure food neophobia in humans in order to identify its antecedents and consequences, as well as effective intervention strategies to reduce it and change unhealthy consumption behavior. Many instruments have been developed for this purpose (for a review, cf. Damsbo-Svendsen, Frøst, \& Olsen, 2017), but the Food Neophobia Scale (FNS, Pliner \& Hobden, 1992) is still the most used measure of food neophobia in adults, probably because it is very specific. Indeed, the other measures are not specifically devoted to quantifying food neophobia, but rather more general or similar constructs (e.g., the Variety Seeking, or VARSEEK, Scale by van Trijp \& Steenkamp, 1992) or a combination of constructs including food neophobia as a subscale (e.g., the Food and Eating Questionnaire by Raudenbush, van der 
1 Klaauw, \& Frank, 1995). The FNS is also the only measure originally validated with a behavioral

2 test, and it has been repeatedly shown to predict actual responses to novel food (e.g., Hobden \&

3 Pliner, 1995; Raudenbush \& Frank, 1999; Raudenbush, Schroth, Reilley, \& Frank, 1998).

4 Furthermore, the FNS is the only food neophobia measure that is completely balanced.

However, the FNS dates back to 1992, and its validation through confirmatory factor analysis dates back to 2003 (Ritchey, Frank, Hursti, \& Tuorila, 2003). For this reason, DamsboSvendsen et al. (2017) suggested that some items in the FNS may no longer be relevant, stating that a novel test of the FNS, focused on the critical assessment of the validity of its items and on the unidimensionality of its structure, should be performed.

This is why, the present study aimed at testing the validity of the FNS in a wide convenience sample of Italian adults. The validity of this scale nowadays was tested going through three steps. First, its construct validity was tested using a confirmatory factor analysis (CFA), and thus the unsatisfactory items have been deleted. A 6-item Revised Food Neophobia Scale (FNS-R) resulted from this initial step. Second, the concurrent validity of the FNS-R was analyzed by taking into consideration the relation with the general neophobia and disgust sensitivity. Indeed, as neophobic individuals tend to display a general reluctance to experience new situations, people and activities (i.e., high level of general neophobia; Pliner \& Hobden, 1992; Raudenbush et al., 1995), and a dispositional inclination to experience the emotion of disgust (i.e., high disgust sensitivity; AlShawaf, Lewis, Alley, \& Buss, 2015; Björklund \& Hursti, 2004; Nordin, Broman, Garvill, \& Nyroos, 2004), positive correlations between the FNS-R and both the General Neophobia Scale (GNS; Pliner \& Hobden, 1992) and the Contamination Disgust subscale of the Revised Disgust Scale (DS-R; Olatunji et al., 2007, 2009) were expected. Finally, as a third step, the structural invariance of the FNS-R across genders, age groups, levels of education, and method of administration (online vs. paper-and-pencil questionnaire)was tested.

\section{Method}

\section{Participants and procedure}


Data for the present study were gathered along with data for other research purposes. Overall,

2711 adults $\left(69.6 \%\right.$ females, aged $18-73$ years, $\left.M_{\mathrm{age}}=34.34, S D=11.90\right)$ took part in this research

3 (an overview of their sociodemographic characteristics is displayed in Table 1). They were recruited

4 mainly through snowball sampling on Facebook, but also through students' mailing lists. In

5 addition, the present data included pre-school children's parents recruited through school principals

6 and teachers. As data from different studies were merged, not all participants completed the same

7 measures, except for the FNS. Every study, however, included some sociodemographic questions

8 (age and gender) and psychological scales (based on the study, participants were asked about their

9 personality, general neophobia, disgust sensitivity, death anxiety, sociopolitical attitudes, parenting

Table 1. Overview of participants' sociodemographic characteristics. others $(n=108)$ filled in a paper-and-pencil questionnaire. styles, and willingness to taste a list of novel foods). The full questionnaires are available from the corresponding author. Most participants $(n=603)$ completed an online questionnaire, whereas the

18-39 years old $\quad 40-73$ years old

\begin{tabular}{lllll}
\hline \multirow{2}{*}{ Education not asked } & Males & 43 & 8 & 51 \\
\cline { 2 - 5 } & Females & 88 & 17 & 105 \\
\hline $\begin{array}{l}\text { Low education } \\
(\leq 13 \text { years })\end{array}$ & Males & 56 & 50 & 106 \\
\cline { 2 - 5 } & Females & 145 & 65 & 210 \\
\hline $\begin{array}{l}\text { High education } \\
(>13 \text { years })\end{array}$ & Males & 32 & 27 & 59 \\
\hline Total & Females & 118 & 62 & 180 \\
\hline
\end{tabular}

\subsection{Measures}

After giving their informed consent, the participants completed the FNS (Pliner \& Hobden, 1992). Respondents were asked to report the extent to which each of the 10 items described them, 
1 using 5 response categories labeled at their extremes as $1=$ not at all descriptive of me and $5=$ very

2 descriptive of me. The items of the original scale and their Italian translations are reported in Table 33 , in the Results section.

In the original scale answers were given on a 7-point agreement scale, but a 5-point scale was preferred in the present study, as analyses based on the Item Response Theory (IRT; e.g., Lambert et al., 2013; Pallant \& Tennant, 2007; Tennant \& Conhagan, 2007) consistently show that using 7 categories leads to the inclusion of non-discriminant response options, thus reducing the validity of the scale (e.g., Roccato, Rosato, Mosso, \& Russo, 2014). In addition, the usual agreement response options was replaced with the above-reported anchors that fit better with the items content and make the questions less ambiguous (Schuman \& Presser, 1981).

A subsample of 448 respondents $\left(73.0 \%\right.$ females, $M_{\text {age }}=34.80$ years, $S D=12.81$, range $=$ 18-73) also filled in the other two scales used to test the concurrent validity of the FNS: the General Neophobia Scale (GNS; 8 items; Pliner \& Hobden, 1992) and the Contamination Disgust subscale from the DS-R (5 items; Olatunji et al., 2007, 2009). Only the latter subscale was administer based on preliminary analyses conducted on a subsample of 264 participants who completed the whole 25-item DS-R, showing that when the three factors (core disgust, animal-reminder, and contamination disgust) of the DS-R were entered as predictors in a linear regression predicting food neophobia, only contamination disgust reached statistical significance $\left(\beta=.29, p<.001, R^{2}=.11\right)$.

Both the GNS and the Contamination Disgust subscale were administered with a 5-category format. For the GNF and the first 2 items of the Contamination Disgust subscale, participants had to report the extent to which each item described them, using the same response scale used for the FNS. For the remaining 3 items of the Contamination Disgust subscale, participants had to rate how disgusting each described situation would be on a 5-response scale labeled at its extremes as $1=n o t$ at all disgusting and 5 = extremely disgusting. Finally, for all participants, a standard sociodemographic form followed, asking about their gender and age $\left(70.3 \%\right.$ females, $M_{\text {age }}=35.71$ 
1 years, $S D=11.79$, range $=18-73$ ). The level of education was asked to 555 participants, and

2 recoded into years of formal education

\section{Data analyses}

The validity of the FNS was analyzed via a threefold procedure. First, its construct validity was analyzed via a confirmatory factor analysis (CFA), performed using AMOS 20.0 (extraction: ML). The scale would have been considered valid only if it was unidimensional. Second, after ascertaining its construct validity, the concurrent validity of the scale was tested via two structural equations models (SEMs) aimed at analyzing its correlation with the GNS (Pliner \& Hobden, 1992) and the Contamination Disgust subscale from the DS-R (Olatunji et al, 2007, 2009). All of these constructs were measured as latent variables, using the items of the questionnaires as their manifest indicators. The scale would have been considered valid only if it showed positive, significant correlations with general neophobia and sensitivity to contamination disgust. The a priori $\alpha$ level to evaluate the significance of these associations was set to 0.05 . The sample size was large enough to conduct a factor analysis on each scale, in that the participants-to-item ratio was much higher than the 12:1 usually considered as the standard threshold (see Byrne, 2012). Consistent with Hu and Bentler's (1998) suggestions, different indexes were combined to evaluate the fit of these models. Based on Schreiber, Stage, King, Nora, and Barlow (2006), the Tucker-Lewis coefficient (TLI: Tucker \& Lewis, 1973), the comparative fit index (CFI: Bentler, 1990), and the root mean square error of approximation (RMSEA: Steiger, 1980) were chosen. Based on Bentler (1990) and Browne (1990), the CFI and the TLI were considered as satisfactory if higher than .90. Moreover, based on Browne and Cudeck (1993), the RMSEA was considered good if lower than 0.05 and fair if ranging between 0.05 and 0.08 . With the exception of the test of the structural invariance of the FNS-R (see below), even if it was reported the $\chi^{2}$ of the models was not taken into consideration, because such an index heavily depends on the $N$ of the dataset.

After ascertaining the validity of the FNS-R, its structural invariance across genders, age groups, levels of education, and method of administration was tested employing Reise, Widaman, 
1 and Pugh's (1993) approach. Starting with gender, a baseline (B) model was tested simultaneously

2 on both gender groups. Subsequently, an invariant (I) model was tested, fixing all of the factor

3 loadings and the correlations between the errors of the con-trait items to be equal among men $(n=$

$4216)$ and women $(n=495)$. The hypothesis of invariance would have been accepted if constraining

5 the parameters to invariance would have not determined a significant worsening in the model fit,

6 i.e., if the difference between the $\chi^{2}$ of the I model and that of the B model was not significant for a

7 number of degrees of freedom equal to the difference in degrees of freedom of the two models. The

8 same procedure was repeated, subdividing the sample into groups based on age (low level = less

9 than 40 years, $n=482$; high level $=$ at least 40 years, $n=229$ ), education (low level $=$ not more than 13 years of formal education, $n=316$; high level = more than 13 years of formal education, $n=$ 239), and method of administration (online: $n=603$; paper-and-pencil: $n=108$ ).

\section{Results}

In spite of the very good alpha of the scale, $\alpha=.89$, a first CFA on the 10-item FNS did not show an adequate fit to the data: $\chi^{2}(35)=335.32, p<.001, T L I=.45, C F I=.49$, RMSEA $=.11$ (90\% CI: .10-.12). Unsatisfactory results such as this may occur even when genuinely unidimensional balanced scales are subjected to confirmatory factor analysis, as a consequence of the partial distortion of the data stemming from response-sets, mainly from acquiescence (cf. Winkler, Kanouse, \& Ware, 1982). To correct for this method distortion, Marsh's (1989) correlated uniqueness approach was employed. This approach states that when a latent variable is measured via a balanced scale, the error variance stemming from response-sets should be statistically controlled for by correlating the errors of the con-trait items. However, even with this correction, the fit of the model was unsatisfactory: $\chi^{2}(25)=286.76, p<.001, T L I=.85, C F I=.92, R M S E A=$ .12 (90\% CI: .11-.13). Nevertheless, the modification indexes showed that the inclusion of items 3 ('If I don't know what a food is, I won't try it'), 4 ('I like foods from different cultures'), 8 ('I am very particular about the foods I eat'), and 9 ('I will eat almost anything') lowered the fit of the 
1 scale. A new analysis, performed after deleting them and correcting for the method factor (i.e.,

2 correlating the errors of the con-trait items), showed that the obtained 6-item scale $(\alpha=.84)$ was

3 definitely unidimensional: $\chi^{2}(6)=30.02, p<.001, T L I=.96, C F I=.99, R M S E A=.07(90 \% \mathrm{CI}$ :

4 .05-.10). All factor loadings were significant with $p<.001$ and, when standardized, ranged between

5.57 and .78 (see Figure 1). The other indexes provided by Amos (available on request) were

6 satisfactory as well. For the sake of brevity, we do not report on them. To conclude, these analyses

7 spoke in favor of the construct validity of the FNS-R.

8

9

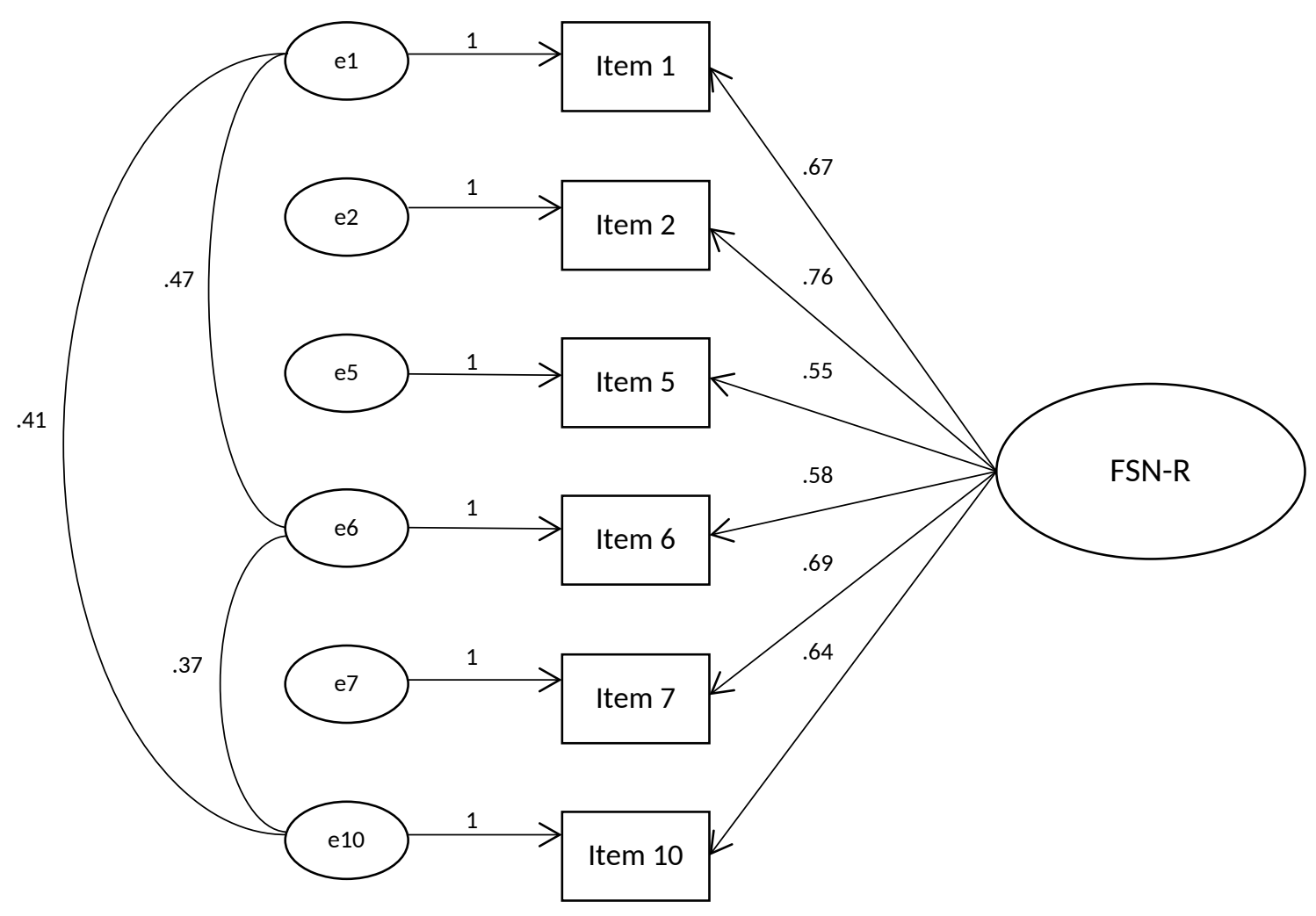

Figure 1. Confirmatory factor analysis of the FNS-R (standardized parameters are displayed). 
1 all $p \mathrm{~s}<.001$, after deleting items 1 ('I feel uncomfortable when I find myself in novel situations'), 2

2 ('Whenever I'm away, I want to get home to my familiar surroundings') and 3 ('I am afraid of the

3 unknown'). The alpha of the resulting battery was satisfactory: $\alpha=.81$.

5 Contamination Disgust subscale did not show a completely satisfactory fit: $\chi^{2}(5)=42.41, p<.001$,

$6 T L I=.94, C F I=.97$, RMSEA $=.10(90 \%$ CI: .08-.13). However, the scale was definitely

7 unidimensional after deleting the item 'As part of a sex education class, you are required to inflate a

8 new unlubricated condom, using your mouth,' $\chi^{2}(2)=3.05, p=.22, T L I=.99, C F I=.99, R M S E A=$ $9.03(90 \% \mathrm{CI}: .00-.08)$, with standardized factor loadings ranging from .47 to .72 , and all $p \mathrm{~s}<.001$. 
1 Table 2. Test of the invariance of the FNS-R across gender, age groups, level of education, and

2 methods of scale administration.

$$
\chi^{2} \quad \text { df } P \quad \text { TLI } \quad \text { CFI } \begin{aligned}
& \text { RMSEA } \\
& (90 \% \mathrm{CI})
\end{aligned} \chi^{2} \text { difference }
$$

\begin{tabular}{|c|c|c|c|c|c|c|c|c|}
\hline $\begin{array}{l}\text { Gender } \\
\text { (men's } n=\end{array}$ & $\begin{array}{l}\text { Baseline } \\
\text { model }\end{array}$ & 41.46 & 12 & .000 & .95 & .98 & $.06(.04-.08)$ & \\
\hline $\begin{array}{l}\text { 216; women's } \\
n=495)\end{array}$ & $\begin{array}{l}\text { Invariant } \\
\text { model }\end{array}$ & 46.62 & 17 & .000 & .97 & .98 & $.05(.03-.07)$ & $\chi^{2}(5)=5.16, p=.40$ \\
\hline $\begin{array}{l}\text { Age groups } \\
(\leq 40 \text { years }\end{array}$ & $\begin{array}{l}\text { Baseline } \\
\text { model }\end{array}$ & 35.66 & 12 & .000 & .96 & .99 & $.05(.03-.07)$ & \\
\hline olds' $n=482$ & & & & & & & & \\
\hline $\begin{array}{l}>40 \text { years } \\
\text { olds' } n=229 \text { ) }\end{array}$ & $\begin{array}{l}\text { Invariant } \\
\text { model }\end{array}$ & 40.40 & 17 & .001 & .97 & .99 & $.04(.03-.06)$ & $\chi^{2}(5)=4.74, p=.45$ \\
\hline
\end{tabular}

\begin{tabular}{|c|c|c|c|c|c|c|c|c|}
\hline $\begin{array}{l}\text { Level of } \\
\text { education }(\leq\end{array}$ & $\begin{array}{l}\text { Baseline } \\
\text { model }\end{array}$ & 18.69 & 12 & .10 & .99 & 1.00 & $.03(.00-.05)$ & \\
\hline $\begin{array}{l}13 \text { years } n= \\
316 ;>13 \\
\text { years } n=239 \text { ) }\end{array}$ & $\begin{array}{l}\text { Invariant } \\
\text { model }\end{array}$ & 22.22 & 17 & .18 & .99 & 1.00 & $.02(.00-.04)$ & $\chi^{2}(5)=3.53, p=.62$ \\
\hline $\begin{array}{l}\text { Methods of } \\
\text { administration }\end{array}$ & $\begin{array}{l}\text { Baseline } \\
\text { model }\end{array}$ & 36.24 & 12 & .000 & .96 & .99 & $.05(.03-.07)$ & \\
\hline
\end{tabular}

603; paper- Invariant

and-pencil $n \quad$ model

$\begin{array}{lllllll}51.24 & 20 & .000 & .97 & .98 & .05(.03-.06) & \chi^{2}(8)=15.00, p=.06\end{array}$

$=108)$ 
Table 3. Original items of the FNS and Italian translation.

\section{I am constantly sampling new and different Assaggio sempre cibi nuovi e diversi (R) foods (R)}

\section{I don't trust new foods $\quad$ Non mi fido dei cibi nuovi}

3. If I don't know what a food is, I won't try it Se non so cosa c'è in una pietanza non la provo

4. I like foods from different cultures (R) Mi piacciono cibi di diversi paesi (R)

5. Ethnic food looks too weird to eat Il cibo etnico sembra strano

6. At dinner parties, I will try new foods (R)

Durante le feste sarei disposto a provare cibi nuovi (R)

\section{I am afraid to eat things I have never had Ho paura di mangiare cibo mai provato \\ before prima}

8. I am very particular about the foods I eat

Per quando riguarda il cibo che mangio mi reputo una persona difficile

9. I will eat almost anything (R) Mangio quasi tutto (R)

\section{I like to try new ethnic restaurants (R) Mi piace provare nuovi ristoranti etnici (R)}

1 Note. 'R' stands for 'reverse item.'

2

3

5 (e.g., Arvola, Lähteenmäki, \& Tuorila, 1999; Rigal, Frelut, Monneuse, Hladik, Simmen, \& Pasquet,

6 2006; Stratton, Vella, Sheeshka, \& Duncan, 2015). However, its psychometric properties needed to

7 be re-examined because of the contextual changes that made new foods much more available and

8 salient than ever. Therefore, the current study aimed to test the validity of the FNS in a wide

9 convenience sample of Italian adults. In addition to testing the construct validity, it aimed at

10 demonstrating concurrent validity through positive relations with the general neophobia orientation 
1 (GNS) and disgust sensitivity (DS-R). Finally, the FNS was expected to be structurally invariant 2 across genders, age groups, levels of education, and methods of administration.

While confirmatory factor analyses revealed mediocre fits for the original 10-item version, a shortened 6-item version (i.e., the FNS-R scale), in which four items with a suboptimal association with the other items of the scale have been deleted and the acquiescent response-set was corrected, displayed a completely satisfactory fit. From the substantive point of view, the four items that lowered the fit of the scale did not appear truly pertinent to food neophobia in the strictest sense. Indeed, items 3 ('If I don't know what a food is, I won't try it'), 8 ('I am very particular about the foods I eat'), and 9 ('I will eat almost anything') could not discriminate between neophobic individuals and picky eaters (who reject a large amount of both familiar and novel foods), vegans/vegetarians, and intolerant/allergic people. Consistent with this, in a large representative sample of Finns, these three items were found to load on a second factor explaining only $7.7 \%$ of the scale variance (Tuorila, Lähteenmäki, Pohjalainen, \& Lotti, 2001). In addition, item 4 ('I like foods from different cultures') refers to liking and thus implies that those foods have already been tasted and are no longer novel, whereas 'the concept of food neophobia only extends to the point where the individual picks up the food and places it in his mouth' (Dovey, Staples, Gibson, \& Halford, 2008, p. 185).

Therefore, the removal of these four items represented an improvement of the measure, as it should be more focused and thus more sensitive to the construct of interest. This is not to say that results previously stemming from the full Pliner and Hobden (1992) scale were no longer valuable. However, as food neophobia is often considered a subset of the picky eating phenomenon (Dovey et al., 2008) and not the reverse, eliminating the potential confusion with pickiness could produce larger magnitude effects. Further research aimed to test this hypothesis will be welcome. In addition, as compared to the FNS, the FNS-R is likely to be more useful for studying the actual interplay between food neophobia and pickiness. Indeed, the issue of picky eating in adulthood, thus far under-investigated, has been recently drawing scholars' attention (Kauer, Pelchat, Rozin, \& 
1 Zickgraf, 2015; Wildes, Zucker, \& Marcus, 2012), and a measure addressing this construct has been 2 newly developed (Zickgraf \& Ellis, 2018).

Overall, the 6-item FNS-R focused on the conceptual core of food neophobia, is fully

4 balanced and an internally consistent, valid, and reliable measure that can be used to assess an

5 individual's orientation toward new food. Moreover, its parameters were invariant according to

6 participants' gender, age, and education, and according to the method used to administer it.

7 Therefore, the FNS-R is preferable to the original FNS for at least four main reasons.

First of all, it has been subjected to more diagnostic and severe psychometric tests on its factorial structure, going well beyond the classic analysis of the scale's $\alpha$ and/or its unidimensionality as stemming from exploratory factor analysis (Pliner \& Hobden,1992; Ritchey et al., 2003).

Second, it displayed a good convergent validity, showing the expected associations with general neophobia and sensitivity to contamination disgust. The fact that the convergent validity of the scale was tested via the SEM approach was definitely a plus of this study, in that it allowed to test the models' fit to the data even when correlating the FNS-R with the other variables taken into consideration.

The third strong point for the FNS-R is related to its length. Indeed, it is shorter than the original scale. The methodological literature converges showing that the longer the scales, the higher the probability of having results distorted by the acquiescent response-set, especially when interviewing samples extracted from the general population and/or in suboptimal settings (e.g., Schuman \& Presser, 1981). It could be objected that a 10-item scale is not that long, but the present analyses showed some symptoms of the acquiescent response-set even in these short scales. Moreover, it is apparent that future researchers will measure food neophobia in association with other variables, thus adding more items. The use of psychometrically solid, as-short-as-possible scales is definitely a fundamental goal of researchers interested in overcoming the 'student sample bias' (Meloen, 1993), according to which substantive research is performed with culturally 
1 privileged student samples, thus undermining the generalizability of the results. The FNS-R

2 definitely goes in that direction.

Finally, the fourth strong point of the FNS-R is related to its structural invariance across

4 participants' main sociodemographic variables and, even more importantly, across methods of

5 administration. It is apparent that the value of a measurement models depends, at least in part, on its

6 replicability across different groups - i.e., if it shows the same psychometric properties across

7 different subsamples, defined according to substantive and/or methodologic criteria (Sass \&

8 Schmitt, 2013). One of the 'new frontiers' of survey research is definitely the web approach, which

9 allows the researcher to interview large samples from the general population at minimum expense

10 in terms of time and money (e.g., Callegaro et al., 2014). The FNS-R was adequate for online

11 administration and thus could be used, even beyond the standard data collection approaches, in web surveys.

A specific comment on the analytical approach used in this paper is germane. Thanks to SEM approach, it was possible to go beyond the often-used, often-insufficiently-diagnostic standard approach, in five senses. First, it allowed to measure the constructs while statistically correcting the random measurement error, thus gaining much more precise estimates of such constructs and of 17 their correlations with other variables. Second, it showed that a scale with a good $\alpha$ needed a 18 relevant trimming of its items to pass the severe fit test of the genuine modeling approach. Third, it allowed to detect some symptoms of distortion stemming from the acquiescent response-set and to correct for it statistically. Fourth, it helped to formally test the fit of the models to the analyzed data . Fifth, and as a byproduct, it allowed to do the first steps for an Italian validation of a short version of the General Neophobia Scale and of the Contamination Disgust subscale of the DS-R.

Beyond its strong points, this research also had some limitations. First, the sample was not statistically representative of the Italian population: Among participants, women and young people were over-represented. In addition, as these data were drawn from different studies, about one-fifth of participants were not asked about their education, thus there was no information on their overlap 
1 with the rest of the sample. However, the invariance across sociodemographic categories suggests

2 that these sample biases should not undermine the FNS-R validity. Nonetheless, a repetition of this 3 study on a more representative sample of the Italian population could be interesting. Moreover, as it 4 systematically happens in Italian research (see Roccato, 2006), socioeconomic status (SES) was not 5 directly measured; instead, education as a proxy of SES was measured, as income-related questions 6 are sensitive issues in Italy, heavily distorted by social desirability. Future research, performed in 7 contexts where people feel freer to declare their social status, such as the USA, will be welcome.

8 However, even before these possible developments, the present study provided a convincing revised 9 version of the most used measure of food neophobia and could be an important reference point for 10 additional research. 


\section{References}

2 Al-Shawaf, L., Lewis, D. M., Alley, T. R., \& Buss, D. M. (2015). Mating strategy, disgust, and food neophobia. Appetite, 85, 30-35.

Arvola, A., Lähteenmäki, L., \& Tuorila, H. (1999). Predicting the intent to purchase unfamiliar and familiar cheeses: the effects of attitudes, expected liking and food neophobia. Appetite, $32(1), 113-126$.

Bentler, P. M. (1990). Comparative fit indexes in structural models. Psychological Bulletin, 107, 238-246.

Björklund, F., \& Hursti, T. J. (2004). A Swedish translation and validation of the Disgust Scale: A measure of disgust sensitivity. Scandinavian Journal of Psychology, 45, 279-284.

Browne, M. W. (1990). MUTMUM PC: User's guide. Columbus: Ohio State University, Department of Psychology.

Browne, M. W., \& Cudeck, R. (1993). Alternative ways of assessing model fit. In K. A. Bollen \& J. S. Long (Eds.), Testing structural equation models (pp. 136-162). Newbury Park, CA: Sage.

Byrne, B. M. (2012). Structural equation modeling with M Plus: Basic concepts, applications, and programming. New York, NY: Taylor \& Francis.

Callegaro, M., Baker, R., Bethlehem, J., Goritz, A. S., Krosnik, J. A., \& Lavrakas, P. J. (Eds.) (2014). Online panel research: A data quality perspective. Oxford: Blackwell.

Damsbo-Svendsen, M., Frøst, M. B., \& Olsen, A. (2017). A review of instruments developed to measure food neophobia. Appetite, 113, 358-367.

Dovey, T. M., Staples, P. A., Gibson, E. L., \& Halford, J. C. (2008). Food neophobia and 'picky/fussy' eating in children: A review. Appetite, 50, 181-193.

Hobden, K., \& Pliner, P. (1995). Effects of a model on food neophobia in humans. Appetite, 25, $101-114$

Hu, L., \& Bentler, P. M. (1998). Fit indices in covariance structure modeling: Sensitivity to undeparametrized model misspecification. Psychological Methods, 3, 424-453. 
1 Kauer, J., Pelchat, M. L., Rozin, P., \& Zickgraf, H. F. (2015). Adult picky eating. Phenomenology, taste sensitivity, and psychological correlates. Appetite, 90, 219-228.

Lambert, S. D., Pallant, J. F., Boyes, A. W., King, M. T., Britton, B., \& Girgis, A. (2013). A Rasch analysis of the Hospital Anxiety and Depression Scale (HADS) among cancer survivors. Psychological Assessment, 25, 379-390.

Marsh, H. W. (1989). Confirmatory factor analyses of multitrait-multimethod data: Many problems and a few solutions. Applied Psychological Measurement, 13, 335-361.

Meloen, J. (1993). The F scale as predictor of fasism: An overview of 40 years of authoritarianism research. In W. F. Stone, G. Lederer, \& R. Christie (Eds.), Strength and weakness: The Authoritarian Personality today (pp. 47-69). New York, NY: Springer.

Nordin, S., Broman, D. A., Garvill, J., \& Nyroos, M. (2004). Gender differences in factors affecting rejection of food in healthy young Swedish adults. Appetite, 43, 295-301.

Olatunji, B. O., Moretz, M. W., McKay, D., Bjorklund, F., de Jong, P. J., Haidt, J., ... \& Page, A. C. (2009). Confirming the three-factor structure of the disgust scale-revised in eight countries. Journal of Cross-Cultural Psychology, 40, 234-255.

Olatunji, B. O., Williams, N. L., Tolin, D. F., Abramowitz, J. S., Sawchuk, C. N., Lohr, J. M., \& Elwood, L. S. (2007). The Disgust Scale: Item analysis, factor structure, and suggestions for refinement. Psychological Assessment, 19, 281-297.

Pallant, J. F., \& Tennant, A. (2007). An introduction to the Rasch measurement model: an example using the Hospital Anxiety and Depression Scale (HADS). British Journal of Clinical Psychology, 46, 1-18.

Pliner, P., \& Hobden, K. (1992). Development of a scale to measure the trait of food neophobia in humans. Appetite, 19, 105-120.

Raudenbush, B., \& Frank, R. A. (1999). Assessing food neophobia: The role of stimulus familiarity. Appetite, 32, 261-271. 
1 Raudenbush, B., Schroth, F., Reilley, S., \& Frank, R. A. (1998). Food neophobia, odor evaluation and exploratory sniffing behavior. Appetite, 31, 171-183.

Raudenbush, B., Van Der Klaauw, N. J., \& Frank, R. A. (1995). The contribution of psychological and sensory factors to food preference patterns as measured by the Food Attitudes Survey (FAS). Appetite, 25, 1-15.

Reise, S. P., Widaman, K. F., \& Pugh, R. H. (1993). Confirmatory factor analysis and item response theory: Two approaches for exploring measurement invariance. Psychological Bulletin, 114, $552-566$.

Rigal, N., Frelut, M. L., Monneuse, M. O., Hladik, C. M., Simmen, B., \& Pasquet, P. (2006). Food neophobia in the context of a varied diet induced by a weight reduction program in massively obese adolescents. Appetite, 46(2), 207-214.

Ritchey, P. N., Frank, R. A., Hursti, U. K., \& Tuorila, H. (2003). Validation and cross-national comparison of the food neophobia scale (FNS) using confirmatory factor analysis. Appetite, 40, 163-173.

Roccato, M. (2006). L'inchiesta e il sondaggio nella ricerca psicosociale [Surveys and polls in social-psychological research]. Bologna: Il Mulino.

Roccato, M., Rosato, R., Mosso, C., \& Russo, S. (2014). Measurement properties of the system justification scale: A Rasch analysis. TPM: Testing, Psychometrics, Methodology in Applied Psychology, 21, 467-478.

Rozin, P. (1976). The evolution of intelligence and access to the cognitive unconscious. Progress in Psychobiology and Physiological Psychology, 6, 245-280.

Rozin, P., \& Millman, L. (1987). Family environment, not heredity, accounts for family resemblances in food preferences and attitudes: A twin study. Appetite, 8, 125-134.

Rozin, P., \& Vollmecke, T. A. (1986). Food likes and dislikes. Annual Review of Nutrition, 6, 433456. 
1 Sass, D. A., \& Schmitt, T. A. (2013). Testing measurement and structural invariance: Implications for practice. In T. Teo (Ed.), Handbook of quantitative methods for educational research (pp. 315-346). Rotterdam: Sense.

Schuman, H., \& Presser, S. (1981). Questions and answers: Experiments on question form, wording, and context in attitude surveys. New York, NY: Academic Press.

Siegrist, M., Hartmann, C., \& Keller, C. (2013). Antecedents of food neophobia and its association with eating behavior and food choices. Food Quality and Preference, 30, 293-298.

Skinner, J. D., Carruth, B. R., Bounds, W., \& Ziegler, P. J. (2002). Children's food preferences: a longitudinal analysis. Journal of the American Dietetic Association, 102, 1638-1647.

Steiger, J. H. (1980). Structural model evaluation and modification: An interval estimation approach. Multivariate Behavioral Research, 25, 173-180.

Stratton, L. M., Vella, M. N., Sheeshka, J., \& Duncan, A. M. (2015). Food neophobia is related to factors associated with functional food consumption in older adults. Food quality and preference, $41,133-140$.

Tennant, A., \& Conaghan, P. G. (2007). The Rasch measurement model in rheumatology: what is it and why use it? When should it be applied, and what should one look for in a Rasch paper? Arthritis Care \& Research, 57, 1358-1362.

Tucker, L. R., \& Lewis, C. (1973). A reliability coefficient for maximum likelihood factor analysis. Psychometrika, 38, 1-10.

Tuorila, H., Lähteenmäki, L., Pohjalainen, L., \& Lotti, L. (2001). Food neophobia among the Finns and related responses to familiar and unfamiliar foods. Food Quality and Preference, 12, 2937.

Van Trijp, H. C., \& Steenkamp, J. B. E. (1992). Consumers' variety seeking tendency with respect to foods: measurement and managerial implications. European Review of Agricultural Economics, 19, 181-195. 
1 Wildes, J. E., Zucker, N. L., \& Marcus, M. D. (2012). Picky eating in adults: Results of a web-based survey. International Journal of Eating Disorders, 45(4), 575-582.

Winkler, J. D., Kanouse, D. E., \& Ware, L. E., Jr. (1982). Controlling for acquiescence response set in scale development. Journal of Applied Psychology, 67, 555-561.

Zickgraf, H. F., \& Ellis, J. M. (2018). Initial validation of the Nine Item Avoidant/Restrictive Food Intake disorder screen (NIAS): A measure of three restrictive eating patterns. Appetite, 123, $32-42$. 
Validation of the revised Food Neophobia Scale (FNS-R) in the Italian context

Margherita Guidettia $^{\mathrm{a}}$, Luciana Carraro ${ }^{\mathrm{b}}$, Nicoletta Cavazza ${ }^{\mathrm{a}}$, Michele Roccato ${ }^{\mathrm{c}}$

aUniversità di Modena e Reggio Emilia, Italy

bUniversità di Padova, Italy

cUniversità di Torino, Italy

Corresponding author: Margherita Guidetti (margherita.guidetti@gmail.com) 